\title{
OPEN Constant output characteristics and design methodology of double side LC compensated capacitive power transfer
}

\author{
Qiao Xiong, Ying Shao ${ }^{\bowtie}$, Pan Sun, Jun Sun, Enguo Rong \& Yan Liang
}

Capacitive power transfer (CPT) has been verified to be capable of transferring a power level as high as inductive power transfer (IPT) recently, and has its own merits. It is a well complement of IPT in near-field wireless power transfer (WPT). This paper gives a newly designed method of realizing both constant output voltage (COV) and constant output current (COC) modes of double side LC compensated CPT. Firstly, through analysis of basic circuit characteristics, the conditions for both of the two modes are deduced theoretically. Especially, one merit of the method is that the conditions indicate a very clear relationship between the compensation components forming resonant tanks. Another merit is that the couple capacitors also participate in resonant tanks. Different from the COV mode, the COC mode can theoretically reach zero phase angle condition simultaneously. Based on these conditions, the parameter design methodology is proposed. Besides, an efficient model of double side LC compensated CPT is built, and the optimum load is calculated theoretically to guide the design course. Finally, the results of both simulations and experiments demonstrate high consistency with the theoretical analysis.

In the past few years, inductive power transfer (IPT) utilizing inductive coils is most widely studied as a conventional wireless power transfer (WPT) technology, though some technical obstacles are difficult to overcome, like bulky couple structure, eddy current loss in nearby conductive objectives, weak performance when misaligned ${ }^{1,2}$. However, capacitive power transfer (CPT) has been verified to be effective and capable of transferring a power level as high as IPT in a significant transfer distance ${ }^{3}$. In 2015, it is reported that CPT can transfer very high power density at $1.1 \mathrm{~W} / \mathrm{mm}^{2}$ at $\mathrm{RF} 100 \mathrm{MHz}$ frequency ${ }^{4}$, and another research reported a prototype that can transfer power in $\mathrm{kW}$ level at $15 \mathrm{~cm}$ transfer distance and high efficiency over $90 \%{ }^{5}$, Since then, increasing attention has been attracted and explosive achievements have been made in CPT research field ${ }^{6}$. Compared with the earlier and more widely studied IPT, CPT has its own merits like flexible and low cost in coupler, good performance when misalignment exists, low eddy current loss induced in nearby metal objects and no requirement for EMI shielding ${ }^{6-8,13,14,19,27}$. Thus, CPT has the potential of applying in some special occasions like in plantable medical appliance $^{9,10}$, rotating machine ${ }^{11}$ or seawater ${ }^{12,13}$. Another breakthrough in CPT technology is that a simplified equivalent model for four-plate coupler was built ${ }^{14}$, based on which further studies of compensation net can be done, to exploit the basic characteristics of a CPT system.

In a CPT system, the significance of compensation net lies in that it can directly define the system transfer property and performance, such as boost the voltage between couple plates to overcome the transfer distance, compensate the system reaction power to make a high power factor, set the voltage or current gain of the system, and consume low energy to ensure a high transfer efficiency. There are plenty of researches focusing on compensation net t $^{5-15-28,30-33}$. The most commonly used compensation nets in CPT range from two-order to four-order. High-order compensation net is often needed to enhance the transfer capability limited by the small value of couple capacitance, like four order compensation net has been adopted ${ }^{15,16}$. However, some defects will be brought about by high-order compensation net, such as increasing the system complexity, high voltage stress on the compensation components, etc. As a result, we would like to further study the transfer property of the 2-order double side LC compensated CPT in this work.

As we know, constant transfer property is one of the basic requirement for a WPT system. A method by changing the operation frequency to achieve constant power and efficiency when the couple situation varies is 


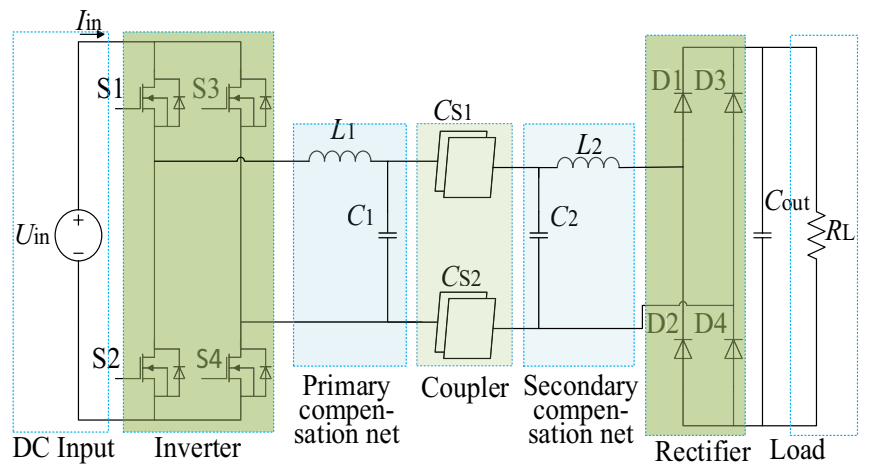

Figure 1. Schematic of a double side LC compensated CPT. (Created by 'Microsoft Office Visio 2013' url: https://www.microsoft.com/zh-cn/microsoft-365/previous-versions/microsoft-visio-2013).

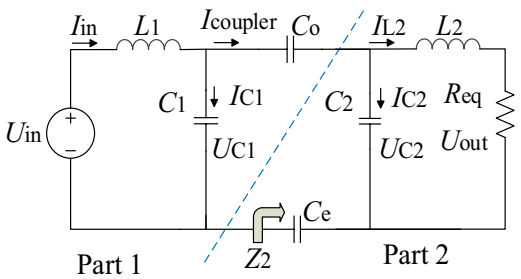

(a)

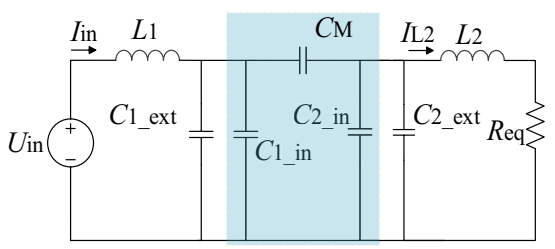

(b)

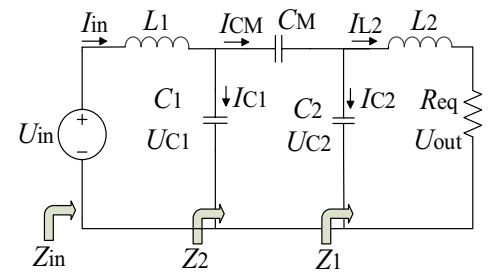

(c)

Figure 2. Equivalent model of CPT with double side LC compensation net. (a) Equivalent circuit with no cross coupling. (b) Simplified circuit model with equivalent coupler model. (c) Further simplified circuit model of (b). (Created by 'Microsoft Office Visio 2013' url: https://www.microsoft.com/zh-cn/microsoft-365/previous-versi ons/microsoft-visio-2013).

proposed $^{17}$, but the close-loop control will increase the complexity and cost of CPT system. In some researches, CPT with double side LC compensation net is analyzed ${ }^{18,19,21,25,30}$, especially both constant output voltage (COV) and constant output current (COC) conditions are proposed based on the deduction of voltage or current gain ${ }^{19}$. However, there are defects in the existing research references. For instance, conditions for both the COV mode and COC mode attribute to operation frequencies different from the intrinsic resonant frequency of the LC compensation net in either the primary side or secondary side, leading to a vague relationship between the resonant components. Furthermore, the couple efficiency in an ordinary CPT system is generally very low, leading to a very vicinal operation frequencies for $\mathrm{COV}$ and $\mathrm{COC}$ modes, which would be also in high accordance with the intrinsic resonant frequency of the LC compensation net. However, the operation frequency can be hardly defined precisely because it is always affected by the manufacture deviation or test deviation of the compensation components and the parasitic reactance of circuit. These will finally induce a trouble in defining the operating frequency in practice. Therefore, this paper aims at disclosing a more intuitive and practical method of decoupling the load resistance, to address the aforementioned problems.

Four categories for constant output are summarized ${ }^{20}$, although they address IPTs, they can also be adopted in CPTs. Accordingly, this paper proposes the COV and COC mode conditions by analyzing the basic circuit characteristics. Then, an efficient model is established and an optimum load resistance is theoretically deduced. Based on these, the design methodology of system parameters is suggested. Both simulation and experiment are carried out to verify them. Finally, three practical issues are discussed. It is demonstrated that the method proposed in this paper is more precise and practical.

\section{Results}

Theoretical model and analysis. A double side LC compensated CPT mainly falls into seven parts, as shown in Fig. 1. Supposing that the input power is a DC source, it should be changed into a high frequency AC that can resonant in the tank of the primary LC compensation net, triggering a high flux electric field between the couple plates, and causing a displacement current from the emission plates of the coupler to the receiving plates. Then, the electricity power going through the coupler will be stored temporarily in the resonant tank of the secondary LC compensation net, providing a source to feed the rectifier and drive the load.

The proposed CPT can be simplified as Fig. 2a. The output of the inverter is treated as a high frequency input voltage in Fig. 2a. When the horizontal distance between plates is significant enough, the cross-couple capacitance can almost be negligible and the couple capacitance is just the capacitance formed by each pair of plates. $C_{\mathrm{o}}$ is defined as the capacitance formed by odd plates $\mathrm{P} 1$ and $\mathrm{P} 3$, and $C_{\mathrm{e}}$ is the capacitance formed by plates $\mathrm{P} 2$ and $\mathrm{P} 4$. When taking consideration of the cross-couple capacitance between each two plates, the equivalent model 


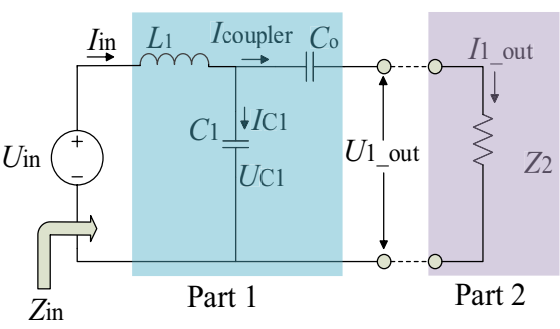

(a)

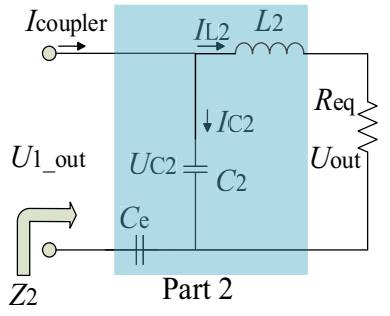

(b)

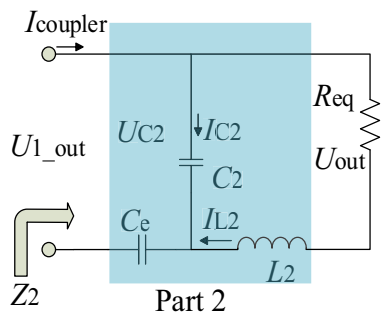

(c)

Figure 3. Symmetric of double side LC compensated CPT and its division of two parts. (a) Part 1 of the circuit, with equivalent resistance of Part 2. (b) Part 2 with output voltage of Part 1 as its input. (c) A change-form of (b). (Created by 'Microsoft Office Visio 2013' url: https://www.microsoft.com/zh-cn/microsoft-365/previous-versi ons/microsoft-visio-2013).

of the coupler is proposed in reference ${ }^{14}$, shown in the blue pane in Fig. $2 b$. The load resistance sourced by the rectifier with a parallel capacitor filter can be equivalent to $R_{\text {eq }}$ by $8 R_{\mathrm{L}} / \pi^{2}{ }^{19}$. In Fig. 2b, suppose $C_{1}=C_{1 \_ \text {ext }}+C_{1 \_ \text {in }}$ and $C_{2}=C_{2 \_ \text {ext }}+C_{2 \_ \text {in }}$. Thus, a further simplified equivalent circuit in Fig. $2 c$ is derived.

Analysis of constant output characteristics. Almost all appliances use electricity as power supply expect a constant input to gain a normal rated power. In this part, the constant output characteristics, including $\mathrm{COC}$ and $\mathrm{COV}$, will be exploited based on the equivalent models given in the former part.

A. COV mode The schematic in Fig. 2a can be divided in two symmetrical parts by the dashed blue line shown in Fig. 3a,b. Figure $3 \mathrm{c}$ is a change form of Fig. 3b, with only a position change in $L_{2}$. Supposing that the operation frequency of the system is $\omega$, the voltage $U_{1 \_ \text {out }}$ in Fig. 3a can be defined by (1), and the voltage $U_{\text {out }}$ in Fig. 3 b can be defined by (2).

$$
\begin{aligned}
U_{1 \_ \text {out }} & =\frac{j \omega C_{\mathrm{O}} Z_{2}}{\Phi_{1}+j \omega C_{\mathrm{O}} Z_{2}\left(1-\omega^{2} L_{1} C_{1}\right)} U_{\text {in }}, \\
U_{\text {out }} & =\frac{j \omega C_{\mathrm{e}} R_{\mathrm{eq}}}{\Phi_{2}+j \omega R_{\mathrm{eq}}\left(C_{2}+C_{\mathrm{e}}\right)} U_{1 \_ \text {out. }} .
\end{aligned}
$$

In (1) and (2), $\Phi_{1}=1-\omega^{2} L_{1}\left(C_{1}+C_{\mathrm{O}}\right), \Phi_{2}=1-\omega^{2} L_{2}\left(C_{2}+C_{\mathrm{e}}\right)$.

Combine (1) and (2), a special voltage gain can be derived by (4) when (3) is met. It is clear that the voltage gain $G_{\mathrm{V}}$ of the CPT has no relationship with $R_{\mathrm{eq}}$ in (4), indicating a constant voltage output of CPT. Thus, Eq. (3) is the condition for COV mode, and it indicates that $L_{1}$ is in resonance with the parallel capacitance of $C_{1}$ and $C_{0}$, and $L_{2}$ is in resonance with the parallel capacitance of $C_{2}$ and $C_{\mathrm{e}}$. The resonant tanks are marked in blue panes in Fig. 3. According to (4), the output power is defined by (5).

$$
\begin{gathered}
\left\{\begin{array}{l}
\Phi_{1}=1-\omega^{2} L_{1}\left(C_{1}+C_{\mathrm{O}}\right)=0 \\
\Phi_{2}=1-\omega^{2} L_{2}\left(C_{2}+C_{\mathrm{e}}\right)=0,
\end{array}\right. \\
G_{\mathrm{V}}=\frac{U_{\text {out }}}{U_{\text {in }}}=\frac{C_{\mathrm{e}}\left(C_{1}+C_{\mathrm{o}}\right)}{C_{\mathrm{o}}\left(C_{2}+C_{\mathrm{e}}\right)}=\frac{\lambda_{1}+1}{\lambda_{2}+1} .
\end{gathered}
$$

In (4), $C_{1}=\lambda_{1} C_{\mathrm{o}}$ and $C_{2}=\lambda_{2} C_{\mathrm{e}}$.

$$
P_{\mathrm{R}_{\mathrm{L}}}=\frac{U_{\text {out }}^{2}}{R_{\mathrm{L}}}=\frac{C_{\mathrm{e}}^{2}\left(C_{1}+C_{\mathrm{o}}\right)^{2} U_{\mathrm{in}}^{2}}{C_{\mathrm{o}}^{2}\left(C_{2}+C_{\mathrm{e}}\right)^{2} R_{\mathrm{L}}}=\left(\frac{\lambda_{1}+1}{\lambda_{2}+1}\right)^{2} \frac{U_{\mathrm{in}}^{2}}{R_{\mathrm{L}}} .
$$

B. In Fig. 2c, resistance $Z_{1}, Z_{2}, Z_{3}$ can be expressed by (6), (7) and (8). Combining (6), (7) and (8), $Z_{2}$ and $Z_{\text {in }}$ can be derived by (9) and (10). To achieve zero phase angle, the input impedance should be purely resistant. In this case, the vector of numerator and denominator in Eq. (10) should have the same angle, a special situation is that the condition in (11) is met. Equation (11) can be changed into (12). From (12), it is easy to find that the inductance $L_{1}$ and a combination of capacitance that $C_{2}$ in serial with $C_{\mathrm{M}}$, then in parallel with $C_{1}$, form a resonant tank in the primary part. Similarly, $L_{2}$ and $C_{1}, C_{2}, C_{\mathrm{M}}$ form another resonant tank in the secondary part. The resonant tanks are exhibited in Fig. 4. The equivalent capacitance of the coupler takes part in both primary and secondary resonant tanks. Substituting (11) into (10), we can get (13). 


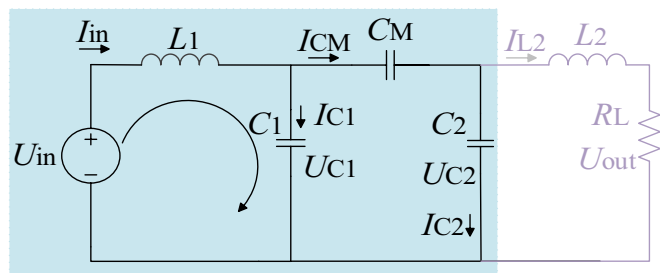

(a)

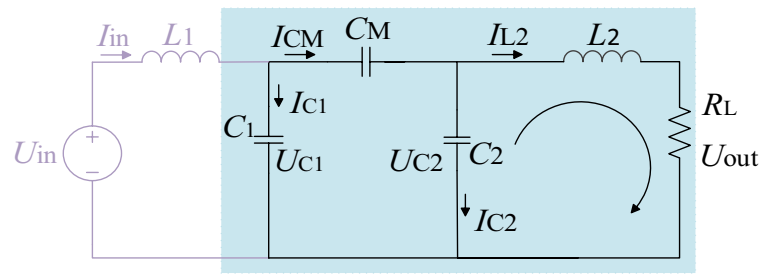

(b)

Figure 4. Resonant tanks in CPT. (a) Resonant tank in primary side. (b) Resonant tank in secondary side. (Created by 'Microsoft Office Visio 2013' url: https://www.microsoft.com/zh-cn/microsoft-365/previous-versi ons/microsoft-visio-2013).

$$
\begin{aligned}
& Z_{1}=\left(j \omega L_{2}+R_{\mathrm{eq}}\right) \| \frac{1}{j \omega C_{2}}=\frac{j \omega L_{2}+R_{\mathrm{eq}}}{1-\omega^{2} L_{2} C_{2}+j \omega R_{\mathrm{eq}} C_{2}}, \\
& Z_{2}=\left(Z_{1}+\frac{1}{j \omega C_{M}}\right) \| \frac{1}{j \omega C_{1}} \\
& Z_{\text {in }}=j \omega L_{1}+Z_{2}, \\
& Z_{2}=\frac{1-\omega^{2} L_{2}\left(C_{2}+C_{\mathrm{M}}\right)+j \omega\left(C_{2}+C_{\mathrm{M}}\right) R_{\mathrm{eq}}}{j \omega \Delta_{2}-\omega^{2}\left(C_{1} C_{2}+C_{1} C_{\mathrm{M}}+C_{2} C_{\mathrm{M}}\right) R_{\mathrm{eq}}}, \\
& Z_{\text {in }}=\frac{1-\omega^{2} L_{2}\left(C_{2}+C_{M}\right)-\omega^{2} L_{1} \Delta_{2}+j \omega R_{\mathrm{eq}} \Delta_{1}}{-\omega^{2} R_{\mathrm{eq}}\left(C_{1} C_{2}+C_{1} C_{M}+C_{2} C_{M}\right)+j \omega \Delta_{2}} . \\
& \text { In (9) and (10), }\left\{\begin{array}{l}
\Delta_{1}=\left(C_{2}+C_{M}\right)-\omega^{2} L_{1}\left(C_{1} C_{2}+C_{1} C_{M}+C_{2} C_{M}\right), \\
\Delta_{2}=\left(C_{1}+C_{M}\right)-\omega^{2} L_{2}\left(C_{1} C_{2}+C_{1} C_{M}+C_{2} C_{M}\right) .
\end{array}\right. \\
& \left\{\begin{array}{c}
\Delta_{1}=0 \\
\Delta_{2}=0,
\end{array}\right. \\
& \left\{\begin{array}{l}
\omega L_{1}=\frac{1}{\omega\left(C_{1}+\frac{C_{2} C_{M}}{C_{2}+C_{M}}\right)} \\
\omega L_{2}=\frac{1}{\omega\left(C_{2}+\frac{C_{1} C_{M}}{C_{1}+C_{M}}\right)},
\end{array}\right. \\
& Z_{\text {in }}=\frac{\left[\omega^{2} L_{1}\left(C_{1}+C_{\mathrm{M}}\right)-1\right] L_{2}}{\left(C_{1}+C_{\mathrm{M}}\right) R_{\mathrm{eq}}} .
\end{aligned}
$$

Then, voltage of $C_{1}, C_{2}$, and $U_{\text {out }}$ can be expressed by (14), (15) and (16), respectively. Combining (6), (13), (14), (15) and (16), the output voltage can be derived from (17). Current of load resistance $R_{\mathrm{L}}$ can be expressed by (18) when $C_{1}=k_{1} C_{\mathrm{M}}$ and $C_{2}=k_{2} C_{\mathrm{M}}$. It can be intuitively seen that the output current of the proposed CPT has no relationship with the load resistance. The power on the load resistance can be defined by (19), which demonstrates that the output power is defined positively by the square of the angular frequency $\omega$ and the load resistance.

$$
\begin{gathered}
U_{\mathrm{C}_{1}}=U_{\text {in }}-j \omega L_{1} \frac{U_{\text {in }}}{Z_{\text {in }}}, \\
U_{\mathrm{C}_{2}}=\frac{Z_{1}}{Z_{1}+\frac{1}{j \omega C_{\mathrm{M}}}} U_{\mathrm{C}_{1}}, \\
U_{\text {Out }}=\frac{R_{\mathrm{eq}}}{R_{\mathrm{eq}}+j \omega L_{2}} U_{\mathrm{C}_{2}}, \\
U_{\text {out }}=\frac{j \omega C_{\mathrm{M}} R_{\mathrm{eq}}}{1-\omega^{2} L_{2}\left(C_{2}+C_{\mathrm{M}}\right)} U_{\mathrm{in}},
\end{gathered}
$$




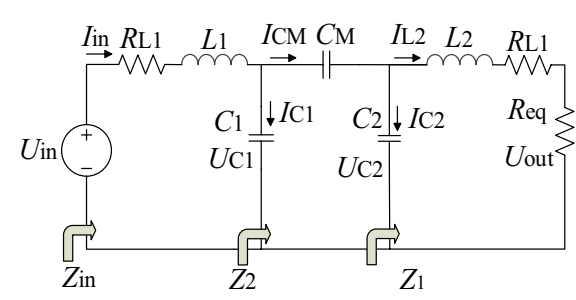

(a)

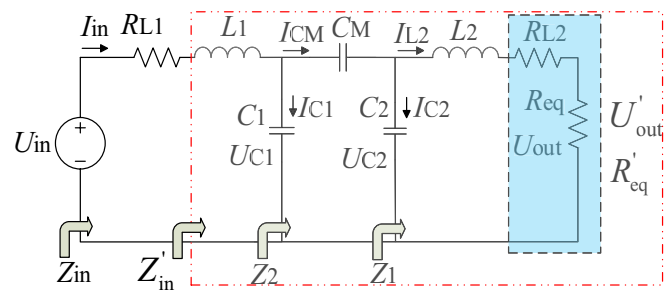

(b)

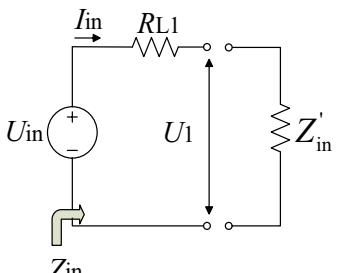

(c)

Figure 5. Efficiency model of COC mode. (a) Circuit with $R_{\mathrm{L} 1}$ and $R_{\mathrm{L} 2 \cdot}$. (b) Replace $\mathrm{R}_{\mathrm{L} 2}$ and $R_{\mathrm{eq}}$ with $R_{\text {eq. }}^{\prime}$ (c) Replace circuit in the red dash line frame in (b) with $Z_{\text {in }}^{\prime}$. (Created by 'Microsoft Office Visio 2013' url: https:// www.microsoft.com/zh-cn/microsoft-365/previous-versions/microsoft-visio-2013).

$$
\begin{gathered}
I_{\mathrm{R}_{\mathrm{L}}}=\frac{U_{\text {out }}}{R_{\mathrm{L}}}=-j \frac{8}{\pi^{2}} \omega\left(\frac{C_{1} C_{2}}{C_{\mathrm{M}}}+C_{1}+C_{2}\right) U_{\text {in }}=-j \frac{8}{\pi^{2}} \omega\left(k_{1} k_{2}+k_{1}+k_{2}\right) C_{\mathrm{M}} U_{\mathrm{in}}, \\
P_{\mathrm{R}_{\mathrm{L}}}=\left(\frac{8}{\pi^{2}}\right)^{2} \omega^{2}\left(k_{1} k_{2}+k_{1}+k_{2}\right)^{2} C_{\mathrm{M}}^{2} U_{\mathrm{in}}^{2} R_{\mathrm{L}} .
\end{gathered}
$$

C. Analysis of $D C-D C$ efficiency From the conclusion above, it is known that the zero phase angle can be achieved in COC mode, making zero voltage switching (ZVS) possible to realize. However, ZVS cannot be achieved in COV mode. Therefore, the COC mode is chosen to analyze the system efficiency. The method for COV mode is similar.

The parasitic resistance of each component in the system is needed to be analyzed when establishing the efficiency model. However, the impact of parasitic resistance from couple capacitors can be limited by choosing high quality factor capacitors $C_{1}, C_{2}$. The non-core inductance winded by litz wire should be adopted to reduce iron-core loss and serial parasitic resistance caused by skin effect when the system works at a high frequency. But a long litz wire will bring non-negligible parasitic resistance. Parasitic resistance also exists in the inverter and rectifier, known as turn-on resistance. In addition, energy loss in the inverter and rectifier also includes switching loss that is much more complex than the turn-on loss, and it has already been well studied ${ }^{34}$. Here, ZVS is supposed to be realized and the switching loss is negligible. Supposing that the parasitic resistance of inverter and $L_{1}$ contributes to $R_{\mathrm{L} 1}$, rectifier and $L_{2}$ contributes to $R_{\mathrm{L} 2}$, shown in Fig. 5a. To simplify the analysis, $R_{\mathrm{L} 2}$ and $R_{\mathrm{eq}}$ can be considered as a whole, $R_{\text {eq }}^{\prime}$. In Fig. $5 \mathrm{~b}$, circuit in the red dash line frame can be treated as an impedance $Z_{\text {in }}^{\prime}$ in Fig. 5c. Based on the assumption above, the system power loss can be calculated by (20). Therefore, the system efficiency can be calculated by (21). The derivation of $\eta$ can be expressed in (22). It can be easily defined that the system efficiency has a maximum optimum value $\eta_{\max }$ when $R_{\text {eq }}^{\prime}$ has the value $R_{\text {eq_op, }}^{\prime}$, calculated by (23).

$$
\left\{\begin{array}{l}
P_{R_{L 1}}=I_{\mathrm{in}}^{2} R_{L 1}=\left(\frac{\omega^{2} \Delta^{2} R_{\mathrm{eq}}^{\prime} U_{\mathrm{in}}}{C_{\mathrm{M}}^{2}+\omega^{2} R_{L 1} \Delta^{2} R_{\mathrm{eq}}^{\prime}}\right)^{2} R_{L_{1}} \\
P_{R_{L 2}}=I_{\text {out }}^{2} R_{L 2}=\left(\frac{8 \omega \Delta}{\pi^{2} C_{\mathrm{M}}} \frac{C_{\mathrm{M}}^{2}}{C_{\mathrm{M}}^{2}+\omega^{2} R_{L 1} \Delta^{2} R_{\mathrm{eq}}^{\prime}} U_{\mathrm{in}}\right)^{2} R_{L 2}=\frac{64 \omega^{2} \Delta^{2} C_{\mathrm{M}}^{2} U_{\mathrm{in}}^{2} R_{L 2}}{\pi^{4}\left(C_{\mathrm{M}}^{2}+\omega^{2} R_{L 1} \Delta^{2} R_{\mathrm{eq}}^{\prime}\right)^{2}},
\end{array}\right.
$$

In (20), $\Delta=C_{1} C_{2}+C_{1} C_{\mathrm{M}}+C_{2} C_{\mathrm{M}}$.

$$
\eta=1-\frac{P_{\text {loss }}}{P_{\text {in }}}=1-\frac{P_{\mathrm{R}_{\mathrm{L} 1}}+P_{\mathrm{R}_{\mathrm{L} 2}}}{P_{\mathrm{in}}}=\frac{C R_{\mathrm{eq}}^{\prime}-B}{A R_{\mathrm{eq}}^{\prime 2}+C R_{\mathrm{eq}}^{\prime}} .
$$

In (21), $\left\{\begin{array}{l}A=\pi^{4} \omega^{2} \Delta^{2} R_{L_{1}} \\ B=64 C_{\mathrm{M}}^{2} R_{L 2} \\ C=\pi^{4} C_{\mathrm{M}}^{2}\end{array}\right.$

$$
\begin{gathered}
\frac{\partial \eta}{\partial R_{\mathrm{eq}}}=-\frac{A C R_{\mathrm{eq}}^{\prime 2}-2 A B R_{\mathrm{eq}}^{\prime}-B C}{\left(A R_{\mathrm{eq}}^{\prime 2}+C R_{\mathrm{eq}}^{\prime}\right)^{2}}, \\
\left\{\begin{array}{l}
R_{\mathrm{eq}_{-} \mathrm{opt}}{ }^{\prime}=\frac{A B+\sqrt{A^{2} B^{2}+A B C^{2}}}{A C} \\
\eta_{\max }=\frac{C^{2}}{2 A B+C^{2}+2 \sqrt{A^{2} B^{2}+A B C^{2}}} .
\end{array}\right.
\end{gathered}
$$




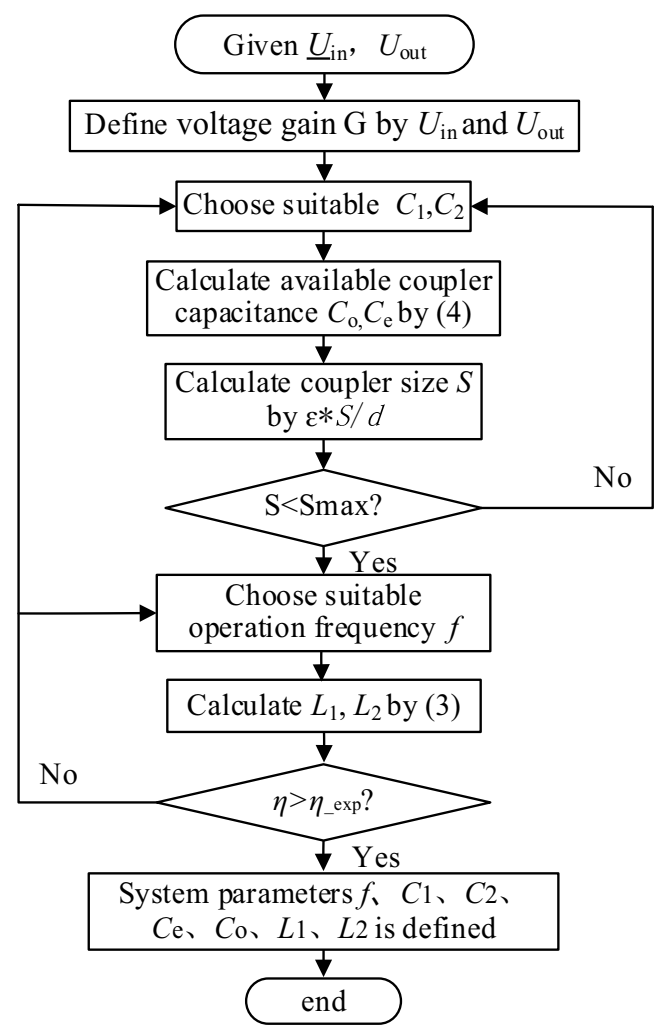

Figure 6. Diagram of parameter design procedure for a COV system. (Created by 'Microsoft Office Visio 2013' url: https://www.microsoft.com/zh-cn/microsoft-365/previous-versions/microsoft-visio-2013).

Design methodology. According to the analysis above, the general design methodology is discussed in this part. Before designing a CPT, the basic demands like nominal input, rated output and physical dimensions allowed for the coupler should be acquired.

A. Parameter design for COV mode If the rated voltage demand $\mathrm{U}_{\mathrm{in}}, \mathrm{U}_{\text {out }}$ is given, the voltage gain is clear. Then, suitable compensation capacitors $C_{1}$ and $C_{2}$ can be chosen. So the coupler capacitance $C_{\mathrm{o}}$, $C_{\mathrm{e}}$ can be defined by (4). Then, the coupler size can be calculated by $\varepsilon^{\star} S / d$. The sizes of the couple plates are often restricted by the available volume of a certain appliance. Thus, to gain a considerable capacitance, a trade-off between the transfer distance and the size of the coupler should be made. If the calculated coupler size is bigger than the allowed range, we should return to choose $C_{1}$ and $C_{2}$. Otherwise, the procedure will continue to choose a suitable operation frequency $f$. Then the value of inductors $L_{1}$ and $L_{2}$ can be calculated. Volume and parasitic resistance of $L_{1}, L_{2}$ is another constraint. Because the parasitic equivalent serial resistance $R_{\mathrm{L} 1}$ and $R_{\mathrm{L} 2}$ is positively related to the value of $L_{1}, L_{2}$. If the efficiency $\eta$ is not higher than the expected or designed value $\eta_{\text {exp }}$, the procedure will go back to choose $C_{1}$ and $C_{2}$, or $f$. After defining $L_{1}, L_{2}$, MOSFETs for the inverter and diodes for the rectifier should be chosen according to the frequency, voltage, and current. The procedure of parameter design for a COV CPT system is concluded in Fig. 6.

B. Parameter design for COC mode The procedure of parameter design for COC CPT system is similar to that of a COV system. However, the difference is that the output current is directly proportional to the angular frequency $\omega$, so when choosing $C_{1}, C_{2}$ and $f$, a trade-off should be made between them. Another trade-off is between the coupler size or transfer distance and the couple capacitance. Especially the output current and power is negatively related to the mutual couple capacitance $C_{\mathrm{M}}$. However, according to the (21), the system efficiency is in positive relationship with $C_{\mathrm{M}}$. A smaller couple capacitance will also require a higher operation frequency or larger resonant inductor, which will trigger a high voltage stress between plates.

These design methodology will be verified in the next section by simulation and experiments. The DC-to-DC efficiency of the system will also be verified.

Simulations and experiments. The constant output characteristics and design methodology are verified through MATLAB simulations and experiment tests. Table 1 gives the parameters designed by the proposed methodology.

Firstly, The system parameters in Table 1 are used to calculate the output characteristics of the system directly in MATLAB. Then, an experiment prototype in Fig. 7 is built for further verification. In this prototype, a DSP board serves as PWM generator, N-Channel SiC MOSFET LSIC1MO120E0080 is adopted to form the full-bridge 


\begin{tabular}{|c|c|c|c|c|c|c|}
\hline \multirow{3}{*}{$\begin{array}{l}\text { Mode } \\
\text { COV }\end{array}$} & \multicolumn{2}{|c|}{ Demands } & \multicolumn{2}{|c|}{$\begin{array}{l}\text { Compensation } \\
\text { components }\end{array}$} & \multicolumn{2}{|c|}{ Coupler size } \\
\hline & $V_{\text {in }}$ & $60 \mathrm{v}$ & $L_{1}\left(L_{2}\right)$ & $33.8 \mathrm{uH}$ & $C_{\mathrm{o}}\left(C_{\mathrm{e}}\right)$ & $2850 \mathrm{pF}$ \\
\hline & $G_{\mathrm{v}}$ & 1 & $C_{1}\left(C_{2}\right)$ & $5.5 \mathrm{nF}$ & $W(l)$ & $265 \mathrm{~mm}$ \\
\hline \multirow{2}{*}{$\mathrm{COC}$} & $V_{\text {in }}$ & $60 v$ & $L_{1}\left(L_{2}\right)$ & $42.8 \mathrm{uH}$ & $d$ & $0.05 \mathrm{~mm}$ \\
\hline & $G_{\mathrm{i}}$ & 0.05 & $C_{1}\left(C_{2}\right)$ & $5.5 \mathrm{nF}$ & $w_{0}$ & $600 \mathrm{~mm}$ \\
\hline \multicolumn{3}{|c|}{ Operation freq $f$} & \multicolumn{2}{|c|}{$300 \mathrm{kHz}$} & $R_{\mathrm{L}}$ & $5 \sim 55 \Omega$ \\
\hline
\end{tabular}

Table 1. Main parameters designed for testing.

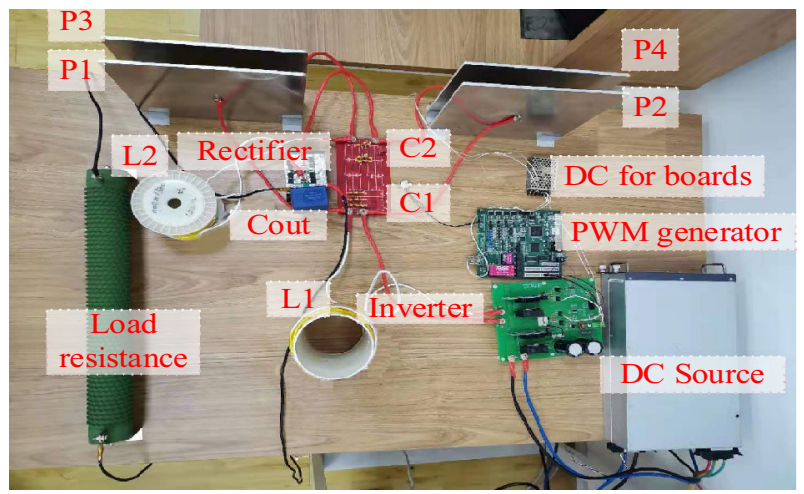

Figure 7. Experiment platform. Taken by the first author Qiao Xiong,through the digital camera of mobilephone, and the descriptive text is added by the software "Microsoft Office Visio 2013" url: https://www. microsoft.com/zh-cn/microsoft-365/previous-versions/microsoft-visio-2013.

\begin{tabular}{|c|c|c|c|c|}
\hline \multicolumn{2}{|c|}{$\begin{array}{l}\text { Component } \\
\text { name }\end{array}$} & Value (pF or $\mathrm{uH})$ & Quality factor & $\mathbf{R}-\mathbf{X}(\boldsymbol{\Lambda})$ \\
\hline \multicolumn{2}{|l|}{$C_{\mathrm{o}}$} & 2849.85 & 954.7 & $0.195-186.16$ \\
\hline \multicolumn{2}{|l|}{$C_{\mathrm{e}}$} & 2858.74 & 672.7 & $0.276-185.67$ \\
\hline \multicolumn{2}{|l|}{$C_{1}$} & 5478.65 & 1126.0 & $0.08600-96.835$ \\
\hline \multicolumn{2}{|l|}{$C_{2}$} & 5493.73 & 1095.9 & $0.08812-96.570$ \\
\hline \multirow{2}{*}{$L_{1}$} & $\mathrm{COV}$ & 33.971 & 587.5 & $0.109+64.034$ \\
\hline & $\mathrm{COC}$ & 42.905 & 680.6 & $0.118+80.306$ \\
\hline \multirow{2}{*}{$L_{2}$} & $\mathrm{COV}$ & 33.829 & 861.7 & $0.074+63.766$ \\
\hline & $\mathrm{COC}$ & 42.8085 & 1871.2 & $0.042+78.592$ \\
\hline
\end{tabular}

Table 2. Numerical quantity tested by LCR meter at $300 \mathrm{kHz}$.

inverter, litz wire is used for all connections in the high frequency part to minimize the loss caused by skin effect. The coupler is made by four aluminum plates with the size shown in the Table 1. Gap distance between each pair of plates is filled with a plastic paper $(0.05 \mathrm{~mm}$ in thickness) to enhance the capacitance of the coupler. In fact, this experiment prototype aims at verifying the constant output characteristics of the suggested system, so the affection from transfer distance is not studied. Numerical quantity of each component in the experiment platform is measured by high-precision LCR Meter, shown in Table 2.

A. COV mode MATLAB simulation results are presented in Fig. 8. It is demonstrated that the system efficiency increases firstly and then decreases with the increase of $R_{\mathrm{L}}$ in Fig. 8a, and the optimum load resistance is about $5 \Omega$. The maximum efficiency is $90.4 \%$, which is in good accordance with the theoretical analysis. Both the amplitude and absolute value of angle of the total input impedance $Z_{\text {in }}$ increase with $R_{\mathrm{L}}$, but the trends become slow. Especially, the angle of $Z_{\text {in }}$ is negative, which indicates $Z_{\text {in }}$ is capacitive, and zero phase angle cannot be achieved. However, the output voltage shows a constant value of about $60 \mathrm{~V}$ when $R_{\mathrm{L}}$ is above $5 \Omega$. Figure $8 \mathrm{~b}$ is the response of the system against the system frequency when $R_{\mathrm{L}}$ is $20 \Omega$. Curves in Fig. $8 \mathrm{~b}$ indicate that the compensation net is intrinsically a band-pass filter. 
Experiment result in Fig. 9a is taken by ZLG Power Analyzer. Due to the voltage drop caused by the parasitic resistance of switch devices, the tested output voltage is slightly lower than simulation. It also shows an DC to DC efficiency of $81.23 \%$ when $R_{\mathrm{L}}$ is $15 \Omega$, close to Fig. $8 \mathrm{a}$. The system efficiency is not so high, due to the significant angle of the total input impedance, and ZVS condition can't be achieved, either. The black curve in Fig. $9 \mathrm{~b}$ shows the output voltage changes with $R_{\mathrm{L}}$, which indicates a tiny increase when the load resistance increasing, due to the decrease of current level in the whole system. The red curve in Fig. $9 \mathrm{~b}$ exhibits that the DC-to-DC efficiency of the COV system decreases with the increase of $R_{\mathrm{L}}$. This phenomenon is in good accordance with the trend of the total input impedance angle shown in Fig. $8 \mathrm{a}$.

B. COC mode Curves drawn by MATLAB in Fig. 10 demonstrate that the total input impedance angle is very close to zero when $R_{\mathrm{L}}$ is more than $5 \Omega$. This means that the zero phase angle and ZVS condition are possible to achieve. The last curve in Fig. 10 shows that the output voltage increase linearly with $R_{\mathrm{L}}$. This means a fine constant output current is achieved. Response of the system when frequency changes in COC mode is similar to the COV mode.

The output waveform of the inverter taken by the oscilloscope is exhibited in Fig. 11. The current waveform slightly lags behind the voltage, indicating ZVS condition is achieved. The DC-to-DC efficiency of the COC system can always reach above $87 \%$. Figure 12 a shows an efficiency of $88.46 \%$ when $R_{\mathrm{L}}$ is $25 \Omega$ and $U_{\text {in }}$ is $58.828 \mathrm{~V}$. The black curve in Fig. 12b indicates the output current is relatively constant when $R_{\mathrm{L}}$ varies and $U_{\text {in }}$ is set to about $58.828 \mathrm{~V}$, and the red curve shows almost the same trend with the simulation results in the first diagram in Fig. 10. The difference between Figs. 10 and $12 \mathrm{~b}$ is mainly because the energy loss of the inverter and the rectifier in Fig. 10 has not been taken into consideration. Experiments have also verified that the system efficiency can easily reach above $90 \%$ when the DC source provides a voltage more than $100 \mathrm{~V}$.

\section{Discussion}

A. Safety issues The electromagnetic safety property of CPT system is often doubted. Some researches ${ }^{9,29}$ have been carried out to investigate it. Nevertheless, safety is a relevant definition. It is undeniable that the electric field in the area between couple plates and in the very nearby area is so high that it may exceed the criterion of the IEEE C95.1 standard ${ }^{35}$. However, the electric field decreases very rapidly with distance ${ }^{19}$. Safety can be ensured except for the dangerous areas. It is pointed out that the dangerous area is within $350 \mathrm{~mm}$ while the couple capacitance is only $2.8 \mathrm{pF}$ and the voltage between plates reaches as high as $1.73 \mathrm{kV}^{19}$. The couple capacitance can be enhanced through many approaches, like coating metallic plates with a very thin high permittivity material, and letting them rely on each other to shorten the distance between plates. Bigger couple capacitance will ensure a lower voltage stress on the plates, thus the dangerous area will be further restricted. Many other approaches can also be applied to ensure safety, like physical isolation to keep the organisms out of dangerous areas, newly designed configuration of the coupler to restrict the power emitted by couple plates.

B. Switch between $\mathrm{COV}$ and COC mode In some cases, switching between COV and COC is required. For example, the course of charging a battery is usually COC first, and COV at the end. According to the proposed decoupling method, the COV and COC system can be designed with a little difference in $L_{1}, L_{2}$ only. Thus, to switch between COV and COC modes, a control loop like Fig. 13 shows can be built. The inductor $L_{1}$ and $L_{2}$ can be divided into two parts in Fig. 13, $L_{1}^{\prime}$ and $L_{2}^{\prime}$ represent the inductance difference between COV and COC modes. The control loop contains a sampler, a tester, a comparator, a driver and two switches $S_{1}, S_{2}$. Suppose CPT system works in COC mode at first, and $S_{1}, S_{2}$ is open. When the voltage reaches a value near the full voltage of the battery, this signal will be sampled and then tested. This tested value will be compared with the predefined voltage point for switching charge mode and then a decision will be made to close $S_{1}, S_{2}$. It should be noted that a communication path needs to be established between the primary and secondary parts to transfer the control signal. Another way to switch between COV and COC mode is to change the operation frequency. Supposing that all compensation components are designed, so the frequency of COV and COC modes can be expressed as (24). When switching between COV and COC mode is required, it is just needed to switch the PWM signal between the frequency $f_{\mathrm{COV}}$ and $f_{\mathrm{COC}}$.

$$
\left\{\begin{array}{l}
f_{\mathrm{COV}}=\frac{\omega}{2 \pi}=\frac{1}{2 \pi \sqrt{L_{1}\left(C_{1}+C_{0}\right)}}=\frac{1}{2 \pi \sqrt{L_{2}\left(C_{2}+C_{e}\right)}} \\
f_{\mathrm{COC}}=\frac{\omega}{2 \pi}=\frac{\sqrt{C_{2}+C_{\mathrm{M}}}}{2 \pi \sqrt{L_{1}\left(C_{1} C_{2}+C_{1} C_{\mathrm{M}}+C_{2} C_{\mathrm{M}}\right)}}=\frac{\sqrt{C_{1}+C_{\mathrm{M}}}}{2 \pi \sqrt{L_{2}\left(C_{1} C_{2}+C_{1} C_{\mathrm{M}}+C_{2} C_{\mathrm{M}}\right)}} .
\end{array}\right.
$$

C. Output stability in response to different compensation parameters and current gain $G_{i}$ It is a common sense that parasitic resistance exists in each component in spite of sparing no effort to reduce it. The parasitic resistance would affect the input or output characteristics of the circuit more or less. The affection by different groups of system parameters and current gain $G_{i}$ is evaluated through MATLAB simulation using the parameters listed in Table 3. The results are shown in Fig. 14. By comparing the curves in Fig. 14, it can be concluded that a smaller $G_{i}$ will induce a more stable output current and efficiency. 


\begin{tabular}{|c|c|c|c|c|}
\hline \multicolumn{2}{|c|}{$\begin{array}{l}\text { Component } \\
\text { name }\end{array}$} & Value (pF or uH) & Parasitic resistance $(\Lambda)$ & Corresponding curve in Fig. 17 \\
\hline \multicolumn{2}{|c|}{$C_{\mathrm{o}}$} & 2849.85 & 0.195 & - \\
\hline \multicolumn{2}{|l|}{$C_{\mathrm{e}}$} & 2858.74 & 0.276 & - \\
\hline \multirow{5}{*}{ Group1 } & $C_{1}$ & 5478.65 & 0.086 & \multirow{5}{*}{ Data 1} \\
\hline & $C_{2}$ & 5493.73 & 0.088 & \\
\hline & $L_{1}$ & 42.905 & 0.118 & \\
\hline & $L_{2}$ & 42.8085 & 0.042 & \\
\hline & $G_{1}$ & 0.0499 & - & \\
\hline \multirow{5}{*}{ Group2 } & $C_{1}$ & 2980.3 & 0.116 & \multirow{5}{*}{ Data 2} \\
\hline & $\mathrm{C}_{2}$ & 3030.6 & 0.099 & \\
\hline & $L_{1}$ & 71.529 & 0.615 & \\
\hline & $L_{2}$ & 70.721 & 0.581 & \\
\hline & $G_{1}$ & 0.0191 & - & \\
\hline \multirow{5}{*}{ Group3 } & $C_{1}$ & 4868.53 & 0.85 & \multirow{5}{*}{ Data 3} \\
\hline & $C_{2}$ & 4685.41 & 0.824 & \\
\hline & $L_{1}$ & 47.393 & 0.32 & \\
\hline & $L_{2}$ & 48.812 & 0.245 & \\
\hline & $G_{1}$ & 0.0397 & - & \\
\hline \multirow{5}{*}{ Group4 } & $C_{1}$ & 1727.14 & 0.065 & \multirow{5}{*}{ Data 4} \\
\hline & $\mathrm{C}_{2}$ & 1714.61 & 0.069 & \\
\hline & $L_{1}$ & 113.34 & 1.288 & \\
\hline & $L_{2}$ & 113.82 & 1.345 & \\
\hline & $G_{1}$ & 0.0085 & - & \\
\hline
\end{tabular}

Table 3. Different groups of compensation parameters.

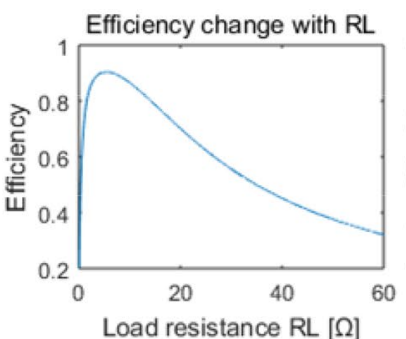

mplitude of Zin change with RL

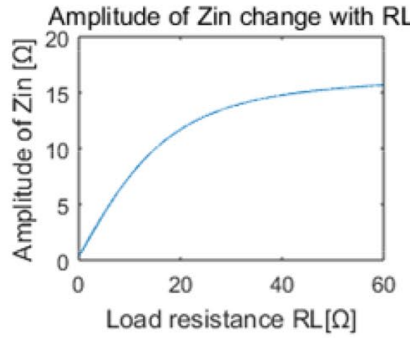

(a)
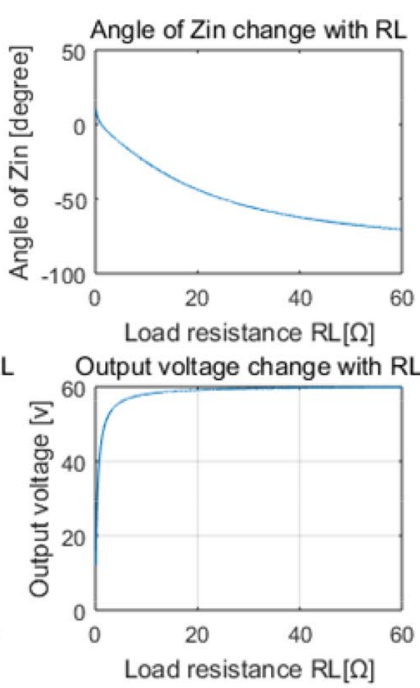
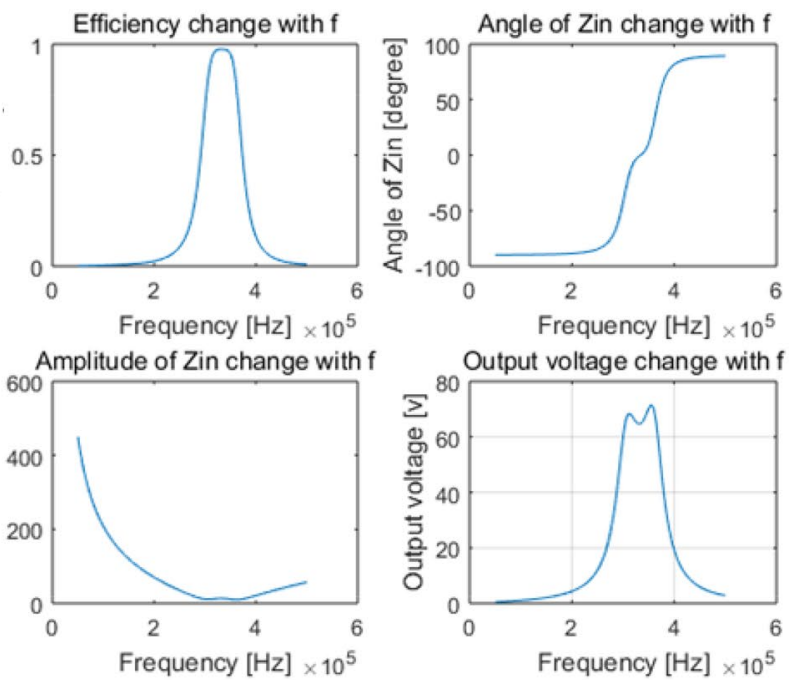

(b)

Figure 8. Main characteristics change with RL (a) and frequency (b) of the proposed system working in constant output voltage mode, derived from MATLAB. (Created by "matlab R2016a" url: https://ww2.mathw orks.cn/products/matlab.html).

\section{Conclusion}

This paper introduces a newly designed load decoupling method that can achieve both COV and COC mode in double side LC compensated CPT. Through the analysis of basic circuit characteristics, the conditions for both two modes are determined. The proposed method has following three advantages: 


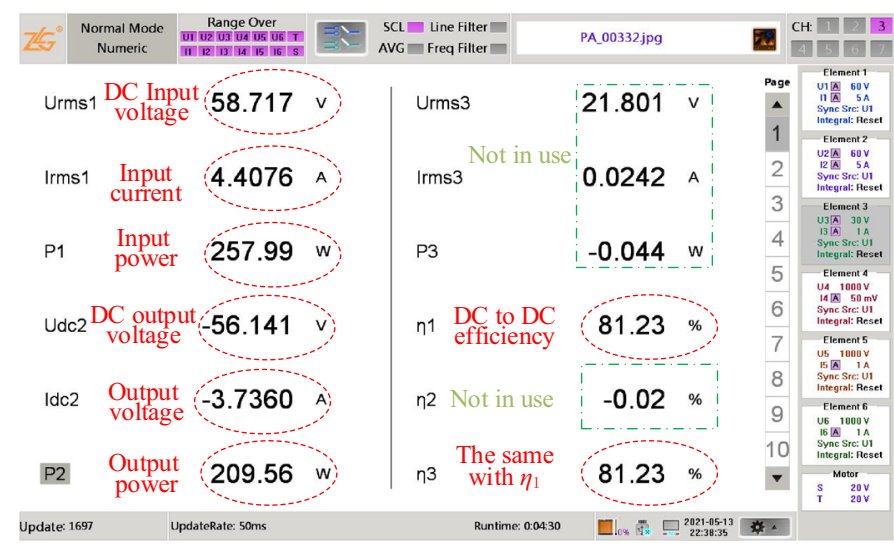

(a)

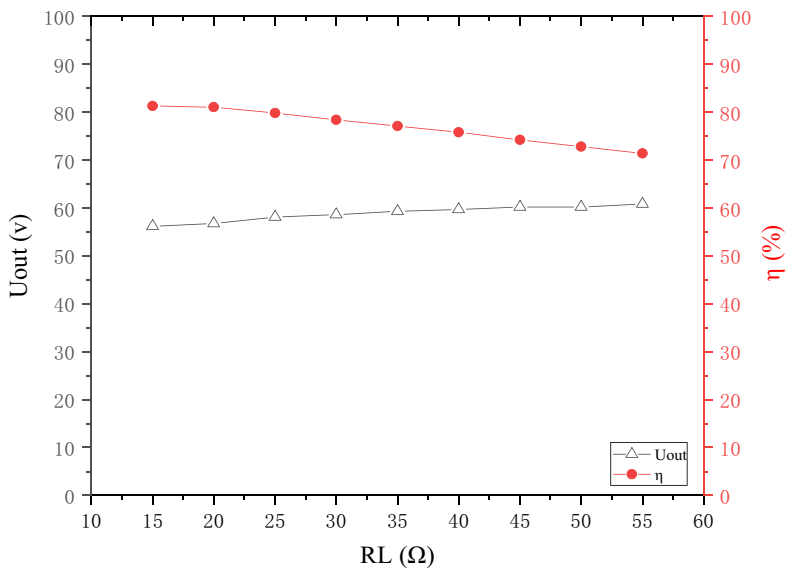

(b)

Figure 9. Experiment result in COV mode. (a) DC input and DC output power tested by ZLG Power Analyzer. (b) The tested output voltage and efficiency change with $R_{\mathrm{L}}$. [(a) is obtained by power analyzer, and the descriptive text is added by the "Microsoft Office Visio 2013" url: https://www.microsoft.com/zh-cn/microsoft365/previous-versions/microsoft-visio-2013, (b) is created by “origin 2018”, url: https:/www.0daydown.com/ tag/originpro-2018].
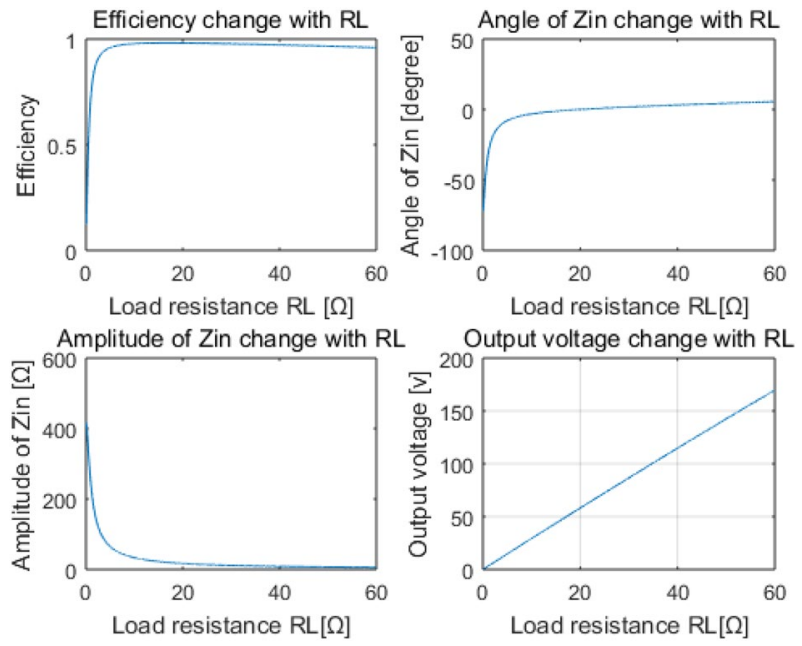

Figure 10. Main output characteristics of the proposed system working in COC mode, derived from MATLAB. (Created by "matlab R2016a" url: https://ww2.mathworks.cn/products/matlab.html).

1. The conditions indicate a very clear relationship between the compensation components;

2. The couple capacitors also participate in the resonant tanks;

3. The COC mode can theoretically reach zero phase angle condition, minimizing the imaginary power as much as possible, while the COV mode can't.

Besides, an efficient model of double side LC compensated CPT is built, and the optimum load is calculated theoretically based on the model. Based on the constant output conditions and efficient model, the parameter design methodology is proposed. Results of both simulations and experiments demonstrate high agreement with the theoretical analysis. Finally, three practical issues are discussed, including electromagnetic safety, switching between the two modes, and stability of output with different groups of parameters. In future research work, we will concentrate on the reduction of parameter sensitivity and optimization of compensation net, efficiency improving scheme and stability control, and the mechanism of transferring power in seawater. 


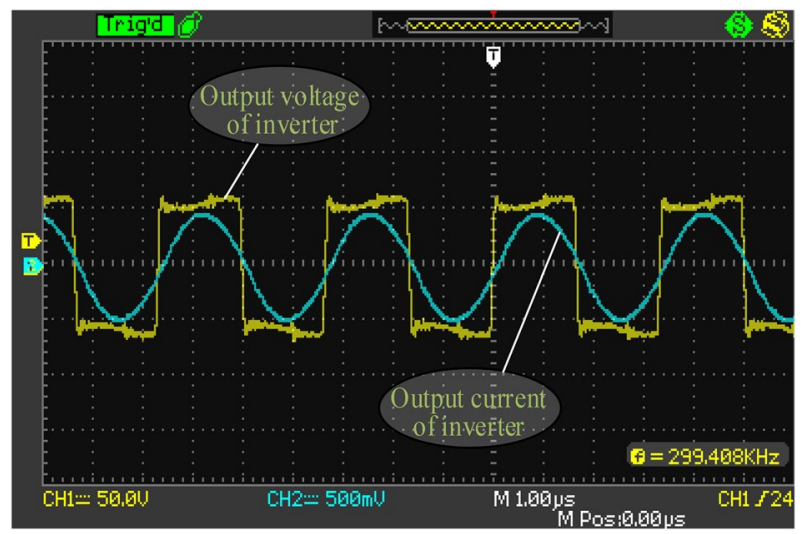

Figure 11. Output waveform of the inverter. (Obtained by oscilloscope, and the descriptive text is added by the "Microsoft Office Visio 2013" url: https://www.microsoft.com/zh-cn/microsoft-365/previous-versions/micro soft-visio-2013).

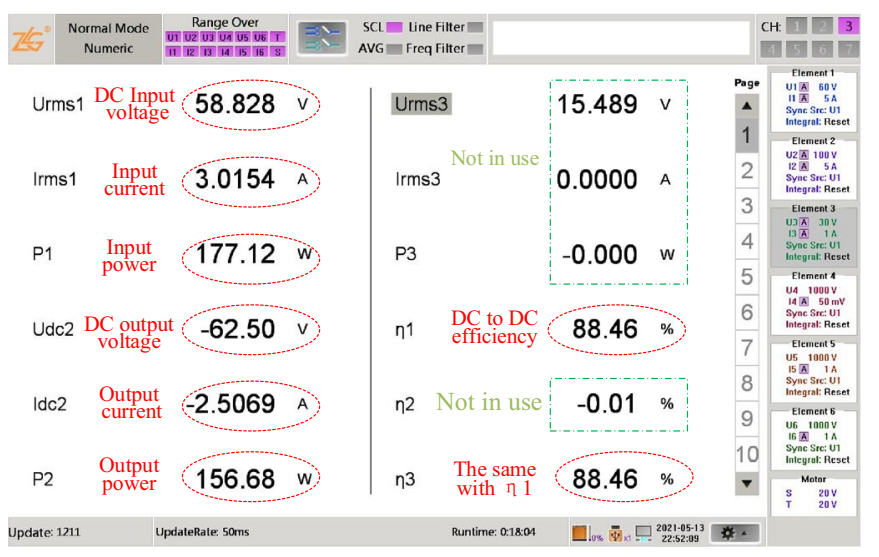

(a)

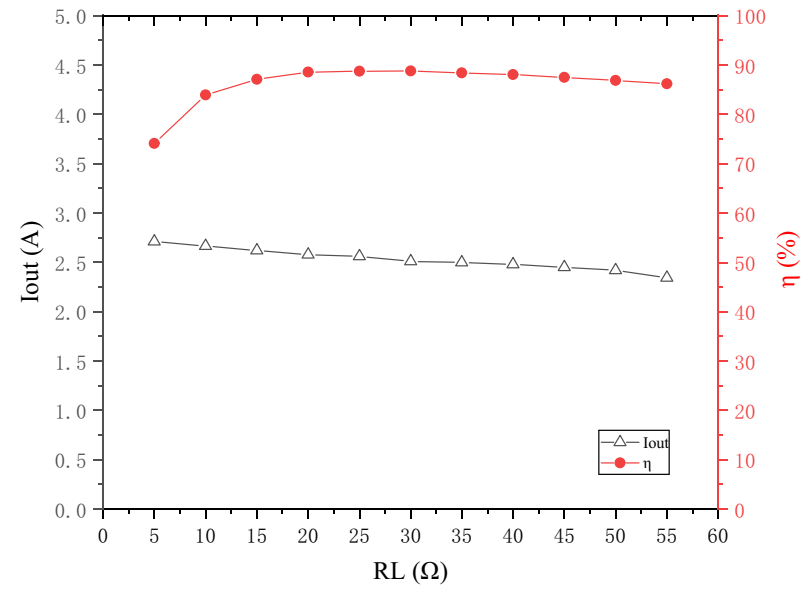

(b)

Figure 12. Experimental results in COC mode. (a) DC input and DC output power tested by ZLG Power Analyzer. (b) The tested output current and efficiency change with RL. ((a) is obtained by power analyzer, and the descriptive text is added by the "Microsoft Office Visio 2013" url: https://www.microsoft.com/zh-cn/micro soft-365/previous-versions/microsoft-visio-2013, (b) is created by origin 2018, url: https://www.0daydown.com/ tag/originpro-2018).

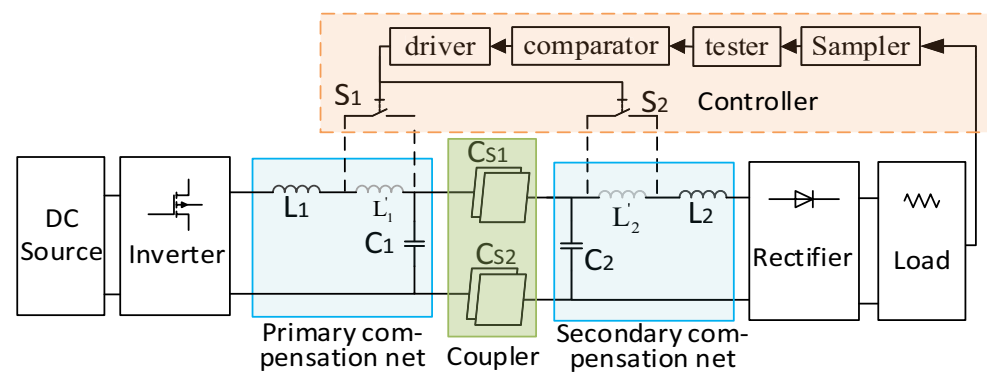

Figure 13. CPT with a control circuit for switching between constant output voltage and COC modes. (Created by the "Microsoft Office Visio 2013" url: https://www.microsoft.com/zh-cn/microsoft-365/previous-versions/ microsoft-visio-2013). 

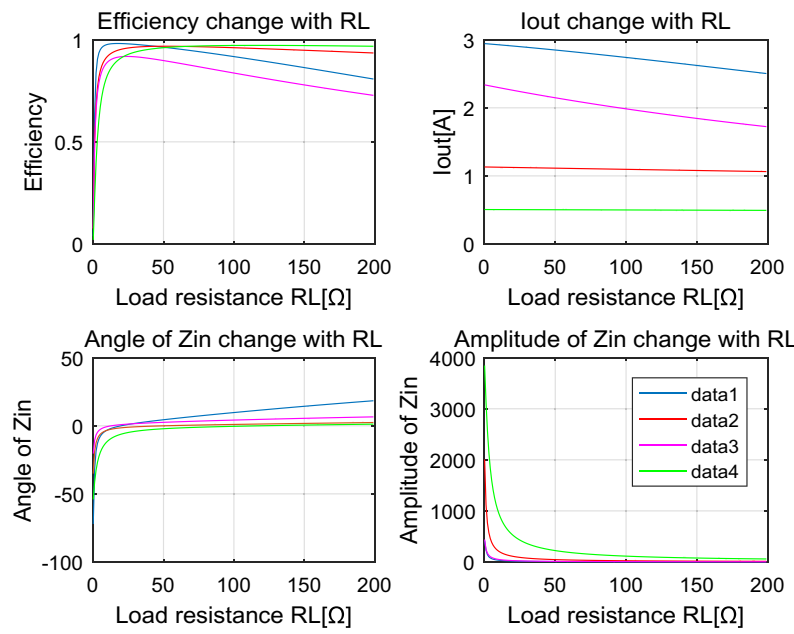

Figure 14. Comparison of output stability when compensated by different parameters or current gain Gi in COC mode. (Created by "matlab R2016a" url: https://ww2.mathworks.cn/products/matlab.html).

Received: 3 December 2021; Accepted: 31 January 2022

Published online: 17 February 2022

\section{References}

1. Pokharel, R. K. et al. Wireless power transfer system rigid to tissue characteristics using metamaterial inspired geometry for biomedical implant applications. Sci. Rep. 11, 5868. https://doi.org/10.1038/s41598-021-84333-3 (2021).

2. Pham, T. S. et al. Optimal frequency for magnetic resonant wireless power transfer in conducting medium. Sci. Rep. 11, 18690. https://doi.org/10.1038/s41598-021-98153-y (2021).

3. Xiaodong, Q. \& Yugang, Su. An overview of electric-filed coupling wireless power transfer technology. Trans. China Electrotech. Soc. 36(17), 3649-3663 (2021).

4. Sepahvand, A. et al. High power transfer density and high efficiency $100 \mathrm{MHz}$ capacitive wireless power transfer system. In Control \& Modeling for Power Electronics IEEE. (2015).

5. Chris, M. High power capacitive power transfer for electric vehicle charging applications. In 6 th International Conference on Power Electronics Systems and Applications (PESA)_Advancement in Electric Transportation-Automotive, Vessel \& Aircraft. (IEEE, 2015).

6. Luo, B. et al. Compensation network design of CPT systems for achieving maximum power transfer under coupling voltage constraints. IEEE J. Emerg. Sel. Top. Power. https://doi.org/10.1109/JESTPE.2020.3027348 (2020).

7. Li, L. T., Wang, Z. P., Gao, F., Wang, S. \& Deng, J. J. A family of compensation topologies for capacitive power transfer converters for wireless electric vehicle charger. Appl. Energy 260(C), 114156 (2020).

8. Liu, Y., Wu, T. \& Fu, M. Interleaved capacitive coupler for wireless power transfer. IEEE Trans. Power Electr. 36(12), 13526-13535. https://doi.org/10.1109/TPEL.2021.3086629 (2021).

9. Al-Kalbani, A. I., Yuce, M. R. \& Redoute, J. M. A biosafety comparison between capacitive and inductive coupling in biomedical implants. IEEE Antennas Wirel. Propag. Lett. 13, 1168-1171 (2014).

10. Khan, S. R. et al. Wireless power transfer techniques for implantable medical devices: A review. Sensors. 20, 3487. https://doi.org/ $10.3390 / \mathrm{s} 20123487(2020)$.

11. Hagen, S., Tisler, M., Dai, J., Brown, I. P. \& Ludois, D. C. Use of the rotating rectifier board as a capacitive power coupler for brushless wound field synchronous machines. IEEE J. Emerg. Sel. Top. Power https://doi.org/10.1109/JESTPE.2020.3039497 (2020).

12. Wu, XSh., Sun, P., Yang, Sh. Q., He, L. \& Cai, J. Review on underwater wireless power transfer technology and its application. Trans. China Electrotech. Soc. 34(08), 1559-1568 (2019).

13. Xiong, Q., Shao, Y., Sun, J., Sun, P., Cai, J. \& Song, X. Y. Analysis for Research achievements and progress trends of underwater electric-field coupled wireless power transfer. In The Proc. 9th Frontier Academic Forum of Electrical Engineering. 742 LNEE, 441-455 (2021).

14. Zhang, H., Lu, F., Hofmann, H., Liu, W. G. \& Mi, C. C. A four-plate compact capacitive coupler design and LCL-compensated topology for capacitive power transfer in electric vehicle charging application. IEEE Trans. Power Electr. 31(12), 8541-8551 (2016).

15. Lu, F., Zhang, H., Hofmann, H. \& Mi, C. A double-sided LCLC compensated capacitive power transfer system for electric vehicle charging. IEEE Trans. Power Electr. 30, 6011-6014 (2015).

16. Lu, F., Zhang, H., Hofmann, H. \& Mi, C. A CLLC-compensated high power and large air-gap capacitive power transfer system for electric vehicle charging application. In IEEE Applied Power Electronics Conference, 1721-1725 (2016).

17. Minnaert, B., et al. Constant capacitive wireless power transfer at variable coupling. 1-4 (2018).

18. Zhang, H. et al. A loosely coupled capacitive power transfer system with LC compensation circuit topology. In 2016 IEEE Energy Conversion Congress and Exposition. (2017).

19. Lu, F., Zhang, H., Hofmann, H. \& Mi, C. C. A double-sided LC-compensation circuit for loosely coupled capacitive power transfer. IEEE Trans. Power Electr. 33(2), 1633-1643 (2018).

20. Yue, R. et al. Constant-voltage and constant-current output using P-CLCL compensation circuit for single-switch inductive power transfer. IEEE Trans. Power Electr. 36(5), 5181-5190 (2021).

21. Sinha, S., Kumar, A., Regensburger, B. \& Afridi, K. K. Design of high-efficiency matching networks for capacitive wireless power transfer systems. IEEE J. Emerg. Sel. Top. Power. 1(1), 992020. https://doi.org/10.1109/JESTPE.2020.3023121 (2020).

22. Kim, D. H. \& Ahn, D. Optimization of capacitive wireless power transfer system for maximum efficiency. J. Electr. Eng. Technol. 15, 343-352. https://doi.org/10.1007/s42835-019-00327-2 (2020).

23. Li, C., Zhao, X., Liao, C. \& Wang, L. A graphical analysis on compensation designs of large-gap CPT systems for EV charging applications. CES Trans. Electr. Mach. Syst. 2(2), 232-242. https://doi.org/10.30941/CESTEMS.2018.00029 (2018).

24. Han, Y. \& Perreault, D. J. Analysis and design of high efficiency matching networks. IEEE Trans. Power Electr. 21(5), 1484-1491. https://doi.org/10.1109/TPEL.2006.882083 (2006). 
25. Xie, S. Y. et al. An electric-field coupled power transfer system with a double-sided LC network. J. Power Electron. 18(1), 289-299 (2018).

26. Su, Y. et al. Parameter optimization of electric-field coupled wireless power transfer system with complementary symmetric LCC resonant network. Trans. China Electrotech. Soc. 34(14), 2874-2883 (2019).

27. Qing, X. D., Wang, Z. H., Su, Y. G., Zhao, Y. M. \& Wu, X. Y. Parameter design method with constant output voltage characteristic for bilateral LC-compensated CPT system. IEEE J. Emerg. Sel. Top. Power. 8(3), 2707-2715. https://doi.org/10.1109/JESTPE.2019. 2936231 (2020).

28. Lu, J. et al. Realizing constant current and constant voltage outputs and input zero phase angle of wireless power transfer systems with minimum component counts. IEEE Trans. Intell. Transport. Syst. 22(1), 600-610. https://doi.org/10.1109/TITS.2020.29856 58 (2021).

29. Su, Y. G., Ma, J. H., Xie, Sh. Y., Zhao, Y. M. \& Dai, X. Analysis on safety issues of capacitive power transfer system. Int. J. Appl. Electromagn. Mech. 53(4), 673-684 (2017).

30. Yi, L. F. \& Moon, J. Y. Design of efficient double-sided LC matching networks for capacitive wireless power transfer system. In 2021 IEEE PELS Workshop on Emerging Technologies: Wireless Power Transfer (WoW) San Diego, USA (2021).

31. Moon, J. High-frequency capacitive wireless power transfer technologies. J. Power Electron. 21, 1243-1257. https://doi.org/10. 1007/s43236-021-00262-4.(2021) (2021).

32. Hou, Y. T. Sinha, S. \& Afridi, K. Tunable multistage matching network for capacitive wireless power transfer system. In 2021 IEEE Wireless Power Transfer Conference (WPTC) San Diego, USA (2021).

33. Kodeeswaran, S. \& Gayathri, M. N. Performance investigation of capacitive wireless charging topologies for electric vehicles. In 2021 International Conference on Innovative Trends in Information Technology (ICITIIT) Kottayam, India (2021).

34. Wu, Y., Chen, Q., Ren, X. \& Zhang, Z. Efficiency optimization based parameter design method for the capacitive power transfer system. IEEE Trans. Power Electr. 36(8), 8774-8785. https://doi.org/10.1109/TPEL.2021.3049474 (2021).

35. IEEE Standard for Safety Levels with Respect to Human Exposure to Electric, Magnetic, and Electromagnetic Fields, $0 \mathrm{~Hz}$ to 300 GHz-Corrigenda 2. IEEE Std C95.1-2019/Cor2-2020, 1-15. https://doi.org/10.1109/IEEESTD.2020.9238523 (2020).

\section{Acknowledgements}

Research work of this paper is supported by 'National Natural Science Foundation of China' under Grant 52007195. The authors would like to thank Pro. Siqi Li, from Kunming University of Science and Technology, China, for his professional guidance of experiments in this paper.

\section{Author contributions}

Q.X. and Y.S. write the main manuscript text and Q.X., J.S., E.R., and Y.L. carry out the experiment tests together. P.S. gives some advises of revision. All authors have reviewed the manuscript.

\section{Competing interests}

The authors declare no competing interests.

\section{Additional information}

Correspondence and requests for materials should be addressed to Y.S.

Reprints and permissions information is available at www.nature.com/reprints.

Publisher's note Springer Nature remains neutral with regard to jurisdictional claims in published maps and institutional affiliations.

(c) (i) Open Access This article is licensed under a Creative Commons Attribution 4.0 International

License, which permits use, sharing, adaptation, distribution and reproduction in any medium or format, as long as you give appropriate credit to the original author(s) and the source, provide a link to the Creative Commons licence, and indicate if changes were made. The images or other third party material in this article are included in the article's Creative Commons licence, unless indicated otherwise in a credit line to the material. If material is not included in the article's Creative Commons licence and your intended use is not permitted by statutory regulation or exceeds the permitted use, you will need to obtain permission directly from the copyright holder. To view a copy of this licence, visit http://creativecommons.org/licenses/by/4.0/.

(C) The Author(s) 2022 